\title{
A LOGÍSTICA HUMANITÁRIA E O PAPEL DAS MÍDIAS SOCIAIS NA COMUNICAÇÃO DE RISCOS DE DESASTRES
}

DOI 10.37619/issn2447-5378.v7i1.333.11-19

\author{
V. L. Monteiro ${ }^{1}, * ;$ M. V. Guimarães ${ }^{2}$; R. A. Moura ${ }^{1}$ \\ 1. Faculdade de Tecnologia de São José dos Campos - Professor Jessen Vidal \\ Av. Cesare Mansueto Giulio Lattes, 1350 - Eugênio de Melo, São José dos Campos/SP, \\ CEP.: 12247-014, Brasil. Telefone: (12) 3905-2423 \\ 2. Instituto Federal de São Paulo - Campus Campos do Jordão \\ Rua Monsenhor José Vita, 280 - Abernéssia, Campos do Jordão/SP, CEP.: 12460-000, Brasil \\ Telefone: (12) 3668-9620 \\ *monteiro vera@uol.com.br
}

\begin{abstract}
RESUMO: Desastres continuam ocorrendo no mundo, levando sociedades à perdas econômicas e de vidas humanas. Com relação às comunicações em situações de risco, sua falta desorienta os afetados, e é de extrema importância para os tomadores de decisão. O objetivo deste artigo foi destacar o papel das tecnologias, como mídias sociais e telefonia móvel, nas comunicações de risco de desastres e propor uma solução que contribua com essa relevante etapa do gerenciamento e resposta aos desastres. A metodologia adotada contou com pesquisa bibliográfica e entrevistas com a Defesa Civil. Como proposta sugeriu-se a criação de um aplicativo georreferenciado, que emita alertas à população e demais envolvidos na resposta à emergência, informando rotas de fuga e localização de abrigos. Concluiu-se que a popularização dos smartphones e sua alta capacidade de processamento faz deles uma alternativa simples e funcional para as comunicações de risco, auxiliando no atendimento às comunidades.
\end{abstract}

PALAVRAS-CHAVE: aplicativos; comunicação; risco de desastre; tecnologias.

ABSTRACT: Disasters continue to occur in the world, leading societies to economic losses and human lives. Regarding to communications in situations of risk, its lack disorients those affected, and it is extremely important for decision makers. The purpose of this article was to highlight the role of technologies, such as social media and mobile telephony, in disaster risk communications and to propose a solution that contributes to this relevant issue of disaster management and response. The methodology adopted included bibliographic research and interviews with the Civil Defense. As a proposal, it was suggested the creation of a georeferenced application, which sends alerts to the population and others involved in the emergency response, informing escape routes and the location of shelters. It was concluded that the popularization of smartphones and their high processing capacity makes them a simple and functional alternative for risky communications, helping to serve communities.

KEYWORDS: applications; communication; disaster risk; technologies.

\section{INTRODUÇÃO}

Os desastres estão se agravando, quer seja pelas alterações climáticas, quer seja pelas ações humanas de urbanização sem planejamento; eles estão aumentando em frequência e intensidade, interferindo negativamente com os objetivos de desenvolvimento sustentável (UNISDR, 2015). 


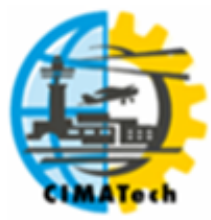

Com o crescimento urbano irregular e as variações climáticas a tendência é que a população mundial sofra cada vez mais com os efeitos de desastres, crescendo o risco de perdas humanas e materiais e, desta forma, aumentando a vulnerabilidade da sociedade (COSTA et al.,2017).

Uma resposta organizada é crucial para mitigar a perda de vidas e os danos à infraestrutura em situações de desastre, sendo que a comunicação é uma das ferramentas fundamentais ao gerenciamento de emergências, tornando-se crucial quando existem dezenas de agências e organizações que respondem, simultaneamente, a um dado evento (SIMON; GOLDBERG; ADINI, 2015).

Ultimamente, as mídias sociais têm sido a principal fonte de informações para o público e também para os responsáveis pelo estabelecimento de uma estratégia de resposta; portanto, a informação fornecida por elas deve ser o mais confiável possível. A crescente popularidade da internet nos últimos anos, vem acarretando a necessidade de se buscar mecanismos para extrair informações úteis e fiéis sobre os eventos de desastres em curso. Para maximizar a eficácia de uma gestão de desastres, a comunicação deve começar cedo, conquistar autoridade entre o público e continuar sendo exercida, regularmente, durante toda a duração de um evento (COLLINS et al., 2016).

Como a possibilidade de interação e a comunicação bidirecional são algumas das principais características das mídias sociais, é razoável supor que elas sejam altamente eficazes no gerenciamento da comunicação de risco (VYNCKE; PERKO; VAN GORP, 2017).

Neste contexto, o objetivo desse artigo de revisão é destacar a importância das novas tecnologias, para uma eficaz troca de informações entre os responsáveis pela resposta em eventos de desastre e o seu público alvo, bem como sugerir uma solução, que atenda às necessidades da Defesa Civil do Estado de São Paulo, e as demais entidades que lidam com a área da Logística Humanitária, de forma a contribuir com seus métodos de comunicação, entre agências, entre seus especialistas, e destes com as comunidades em áreas de risco.

\section{FUNDAMENTAÇÃO TEÓRICO}

Para embasar este artigo de revisão sobre as comunicações de risco e o papel das mídias sociais na Logística Humanitária, este tópico apresenta uma pesquisa bibliográfica recente, nos temas: gestão de riscos de desastre, importância das comunicações na gestão de riscos, o papel das mídias sociais na comunicação de riscos e o uso de aplicativos para dispositivos móveis na comunicação em desastres.

\subsection{Gestão de riscos de desastre}

Segundo o UNISDR (2015) "Risco é o produto das interações entre perigos naturais ou induzidos pelo homem e as condições vulneráveis, incluindo a probabilidade de ocorrência de fenômenos de risco".

Freitas et al. (2012) destacam que os desastres são produzidos socialmente e que a vulnerabilidade das sociedades está relacionada ao seu nível de desenvolvimento econômico e social.

Sendo assim, os desafios da administração de riscos e de desastres exigem a construção de um caminho que incorpore a gestão de riscos ao ordenamento territorial; desenvolvimento urbano; saúde; meio ambiente; mudanças climáticas; gestão de recursos hídricos; geologia; infraestrutura; educação; ciência e tecnologia; e às demais políticas setoriais, tendo em vista a promoção do desenvolvimento sustentável (CEPED-UFSC, 2014). 


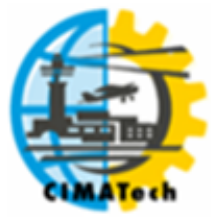

Pohlmann, Piccinini e Da Silva Filho (2014) definem gerir riscos como sendo solucionar problemas para a melhoria da qualidade de vida das sociedades e assim, obter-se reduções em termos de perdas materiais e de vidas.

As estratégias para gestão de riscos devem ainda conter ações para aumentar a capacidade das comunidades, de forma a reduzir suas ameaças e vulnerabilidades, incluindo estratégias que vão além da assistência e da recuperação pós-desastre (OLIVEIRA e ROBAINA, 2015).

Além disso, é importante ressaltar que as comunicações e consultas aos envolvidos na gestão dos riscos é fundamental, pois cada um elabora julgamentos sobre riscos baseado em suas próprias percepções, que estão associadas às diferenças de valores, conceitos e inquietações (HENRIKSEN et al., 2018).

A Política Nacional de Proteção e Defesa Civil (BRASIL, 2012) enfatiza a prevenção dos riscos e incentiva as ações articuladas entre vários setores, com políticas públicas de saúde, de educação, de assistência social, de uso e ocupação do solo, de saneamento básico etc. Neste sentido, as ações de prevenção baseadas na comunicação de riscos, com o intento de compartilhar informações sobre eventos em potencial são fundamentais para uma gestão de riscos eficaz (MARTINS; SPINK, 2015).

\subsection{Importância das comunicações na gestão de riscos}

Situações de desastre são críticas, tornam-se períodos de incerteza que causam estresse coletivo, como resultado da privação da sociedade de suas condições normais de rotina, inclusive da perda de seus meios de comunicações (SIMON; GOLDBERG; ADINI, 2015).

A Informação e a comunicação exercem um papel de extrema relevância nas atividades da logística humanitária. Além disso, é importante destacar que a comunicação tem também uma função educativa e, é dever da administração pública e um direito dos cidadãos (HASELKORN e WALTON, 2009).

Sistemas de monitoramento e alerta, bem como a comunicação de risco, são cruciais para a sobrevivência e recuperação das populações afetadas por desastres. Várias ferramentas podem capacitar as comunidades para agirem em resposta a um alerta de risco e, desta forma, contribuírem com a capacidade das instituições locais de resposta. Percebe-se, então, que a comunicação na gestão de risco pode apoiar não apenas a resiliência e o processo de "recuperação", mas também pode incluir o "como reconstruir melhor", na fase de reconstrução da infraestrutura e do ambiente. Reforçando ainda esse fato, iniciativas recentes na Europa e em países nórdicos, sobre sistemas de alerta e monitoramento, sugerem que é muito positiva a participação de cidadãos e não profissionais ao lidar com os riscos. O acesso aos dados e modelos, por meio da internet, como parte dos sistemas de monitoramento e alerta e de comunicação de riscos, desempenha um papel crucial na recuperação de populações afetadas por desastres (HENRIKSEN et al., 2018).

Assim, aumentar a participação da população na redução de riscos de desastres é uma ação oportuna, já que coloca a atenção no risco, em como os riscos serão entendidos e percebidos pelas pessoas e como as pessoas estão envolvidas em ações de proteção (TYSHCHUK e WALLACE, 2018).

As condições para o gerenciamento das comunicações e para a prevenção de desastres serão tanto melhores quanto melhores forem os recursos tecnológicos disponíveis. Usando a internet é possível interligar redes de sensores, atuadores e big data, além de relatos e históricos de eventos, em computadores interconectados, o que permite ganhos em eficiência, para uma boa gestão de riscos. Diferentes departamentos podem ser interligados, com comunicação via satélite e inteligência artificial, para rastrear ameaças de desastres, bem como, para definir a melhor e mais viável forma de procedimentos de resgate (Figura 1). Um exemplo disso foi o grande terremoto no Japão Oriental, em 2011, que teve seus impactos minimizados graças à eficiência das comunicações, com auxílio das 
novas tecnologias da informação (JOSEPH et al., 2018; JOHN WELLINGTON e RAMESH, 2018; MURAYAMA e YAMAMOTO, 2019).

Assim, a internet deu origem a grandes oportunidades para a comunicação de riscos; ela aboliu a função de controle da mídia tradicional e ofereceu a especialistas não convencionais, uma plataforma para distribuição de informações ao público, o qual pode interagir com esses especialistas e entre si mesmos (VYNCKE; PERKO; VAN GORP, 2017).

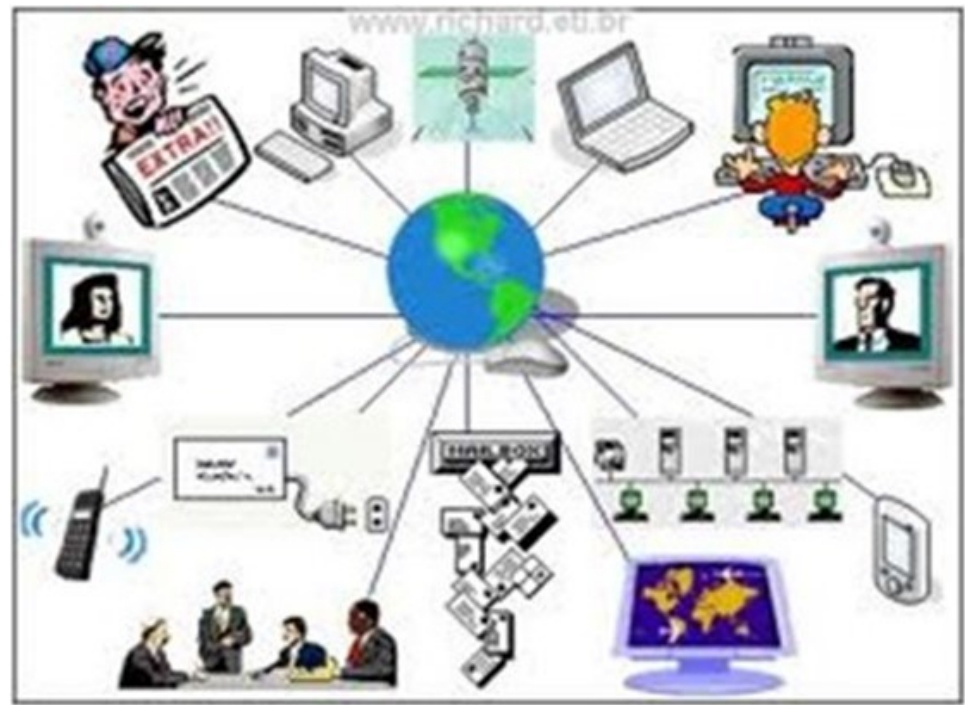

Figura 1. Rede de Informação Interconectada via internet.

\subsection{Papel das mídias sociais na comunicação de riscos}

As mídias tradicionais, como televisão, rádio e jornais, não são mais as principais fontes de informação, pois smartphones e tablets permitem acesso móvel imediato às plataformas de mídia social (COLLINS et al., 2016).

Mídias sociais são canais de relacionamento, baseados na web 2.0, que permitem conexão e interação entre os usuários, mediante o compartilhamento de conteúdos (Figura 2). Também conhecidas como redes sociais, as mais populares atualmente são Facebook, Youtube, Instagram e WhatsApp (LORENZO, 2013; REUTER, 2017).

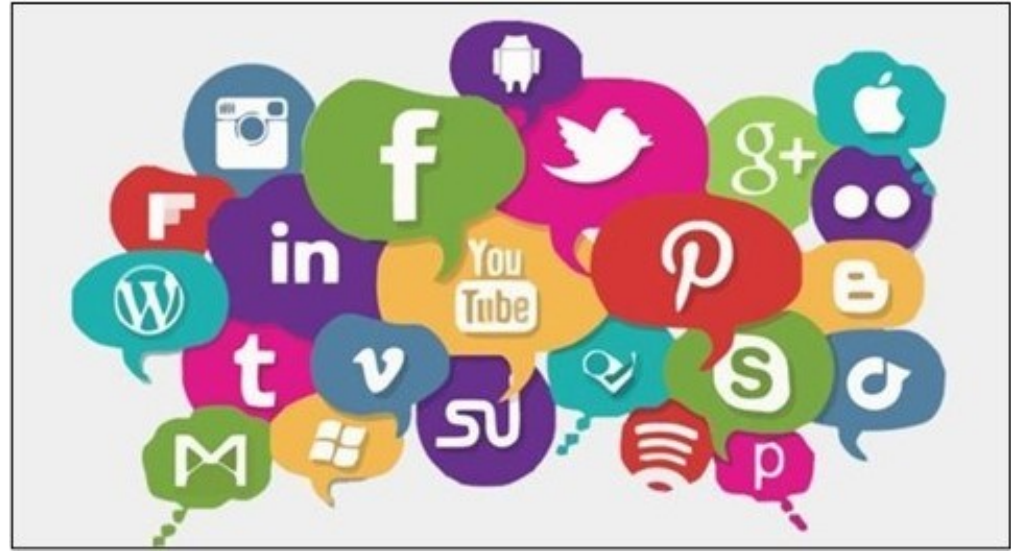

Figura 2. Exemplos de mídias sociais. 


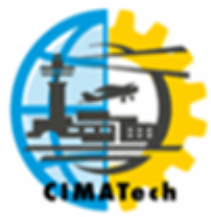

Então, as mídias sociais oferecem oportunidades para envolver os cidadãos na gestão de emergências, disseminando informações ao público e acessando informações deles. Durante eventos de emergência, os indivíduos são expostos a grandes quantidades de informações sem estarem cientes de sua validade ou risco de desinformação, mas os usuários geralmente são rápidos em corrigi-los, tornando a mídia social "autorregulada" (SIMON; GOLDBERG; ADINI, 2015).

As mídias sociais e digitais possibilitam ainda que as comunidades se preparem para os desastres, por meio do compartilhamento de informações, difundidas por dispositivos com tecnologia móvel. O uso das mídias sociais, atende prontamente a divulgação dos boletins, relatórios e protocolos discutidos e planejados e vem se tornando uma prática comum, pois elas, com seu efeito informativo, têm um alcance quase exponencial, graças às tecnologias móveis (WANG; ZHUANG, 2018).

Assim, mídias sociais fazem parte do dia-a-dia de pesquisadores e comunidades e, cada vez mais, tornam-se ferramentas significativamente úteis ao gerenciamento das comunicações nas crises, já que elas disseminam facilmente as informações ao público, e também, possibilitam que haja o compartilhamento de informações pelos cidadãos (SIMON; GOLDBERG; ADINI, 2015).

Juntamente com os benefícios óbvios, incluindo a entrega rápida e eficaz de mensagens, é importante considerar os desafios decorrentes das mídias sociais. A comunicação em crises e desastres deve utilizar a capacidade das mídias sociais para criar um ciclo monitorado de informações instantâneas. A confiabilidade do conteúdo da mídia social, no entanto, é questionável e, portanto, precisam ser desenvolvidas ferramentas de comunicação que possam ser usadas no monitoramento contínuo do cenário, para reduzir o tempo gasto na leitura de mensagens e informações irrelevantes, separando-as de fontes não confiáveis (COLLINS et al., 2016).

\subsection{Uso de aplicativos para dispositivos móveis na comunicação em desastres}

Os avanços nas tecnologias da comunicação, com o surgimento dos smartphones ou telefones inteligentes, facilitou o modo de nos comunicarmos, trabalharmos e nos relacionarmos em sociedade. Essas facilidades, aliadas à diversidade de modelos e preços, tornaram os dispositivos móveis mais acessíveis e populares. Com isso, nasceu também o desenvolvimento de aplicativos para esses celulares, área que é altamente competitiva, tem elevados níveis de exigência e ainda, tem que lidar com diversos aspectos, visando a plena satisfação dos clientes (NASCIMENTO JÚNIOR, 2017).

Os aplicativos para dispositivos móveis podem se tornar muito úteis em situações de desastres ou emergências, tendo em vista que, de acordo com a CISCO (2020), haverá cerca de 29,3 bilhões de dispositivos em rede até 2023. Os smartphones tornaram-se tão populares que deixaram de ser artigos de luxo acessível à poucos, para tornarem-se itens de necessidade básica, presentes hoje na vida de grande parte da sociedade (OLIVEIRA; SANTOS, 2018).

Potencialmente, muitos usuários da Internet podem relatar eventos por meio de seus dispositivos móveis, com muito mais rapidez do que agências de notícias ou especialistas oficiais. Embora uma série de dados como informações geográficas exijam altíssima precisão e qualidade, a experiência sugere que informações adequadas, e não necessariamente de alta qualidade, são suficientes no campo da gestão de crises e operações humanitárias (VAHIDNIA; HOSSEINALI; SHAFIEI, 2020).

De acordo com Lv; Liao e Deng (2018) em uma situação de desastre, onde um grupo de pessoas fica preso sobre escombros, ou soterrado, ou simplesmente isolados devido a um evento extremo, uma rede baseada em tecnologia móvel (posicionamento e comunicação móvel via celular), posicionamento por satélite e tecnologias de cena geográfica $3 \mathrm{D}$, podem ser extremamente úteis para aumentar a capacidade de ação e apoiar uma rápida tomada de decisão dos órgãos responsáveis pelos resgates. 


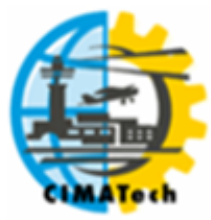

Desta forma, os dispositivos móveis colaboram com a acessibilidade e quebram barreiras temporais, bem como viabilizam comunicações em rede, tornando-se estratégicos na comunicação e prevenção de riscos de desastres. Entretanto persiste uma lacuna no que diz respeito à oferta de aplicativos móveis para comunicação em desastres, para auxílio às populações vulneráveis a desastres (DA SILVA; STEINBERGER-ELIAS, 2015).

\section{ANÁLISES E CONSIDERAÇÕES FINAIS}

A popularização dos dispositivos móveis de comunicação, com alta capacidade de processamento de dados e com preços acessíveis ao grande público, trouxe uma revolução para a coleta remota de dados, e desta forma, para as operações de comunicação em eventos de desastre. Enquanto anteriormente, agentes de ações humanitárias utilizavam lápis e papel para as anotações em campo, hoje temos uma imensa variedade de ferramentas digitais, que são executadas em smartphones, capturando imagens e demais informações, em tempo real, e disponibilizando aos tomadores de decisão.

Nossa sociedade hoje é dependente desses dispositivos móveis, os quais se configuram como alternativas estratégicas frente ao cenário atual e podem contribuir com a promoção da educação e conscientização dos riscos aos quais as sociedades estão expostas. Entretanto, o uso de tecnologias móveis como forma eficaz de troca de informações, ainda enfrenta desafios tais como: rede móvel ou $w i$ - $f i$ não disponíveis em alguns ambientes e/ou situações, informações manipuladas, não confiáveis ou falsas etc. Contudo avanços estão previstos como na infraestrutura de base e nas fontes de energia, de forma a conectar cada vez mais pessoas em todos os lugares do planeta.

Tendo esse contexto sido exposto, a proposta dos autores para incrementar a comunicação de risco em áreas de desastres, é o desenvolvimento de uma aplicativo georreferenciado, onde mapas das áreas de risco possam ser inseridos pelos municípios e por onde seja possível emitir alertas à todos os cidadãos que se encontrem nessas áreas, fornecendo inclusive as opções de rotas de fuga a serem utilizadas e quais abrigos receberão os desabrigados. Esse aplicativo também enviaria mensagens aos responsáveis pelos abrigos, e simultaneamente, aos demais organizadores de tarefas como recolhimento de doações, triagem etc., e para reunir voluntários. Seria interessante ainda, que os moradores da área em risco pudessem enviar mensagens e fotos para as agências humanitárias envolvidas na operação de resposta, a fim de informar a situação vivenciada, como fotos do evento em andamento em tempo real. Porém, o compartilhamento dessas fotos e informações, com os demais cidadãos, seria tarefa apenas dessas agências gestoras do aplicativo, a fim de não acontecer que falsas notícias e/ou notícias geradoras de pânico ou manipuladoras, circulem entre os atingidos. Além disso tudo, o aplicativo pode ainda servir para o rastreamento das equipes em ação, de forma a garantir a segurança de todos, mediante conhecimento de sua localização e situação, em tempo real.

\section{CONCLUSÕES}

A proposta deste estudo foi destacar a importância das comunicações e o potencial das novas tecnologias para auxiliar a Defesa Civil e demais agências de ajuda humanitária, nas comunicações de risco de desastre e ainda, propor uma nova solução para a comunicação, que possa ser utilizada nos alertas de risco, bem como, para receber informações das comunidades afetadas. Para tanto, os principais métodos de pesquisa adotados foram: entrevistas com agentes da Defesa Civil do Estado de São Paulo, combinadas com análises de conteúdo de documentos oficiais relevantes. 


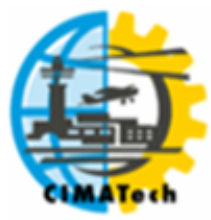

O aplicativo georreferenciado proposto está de acordo com o contexto social atual, onde as tecnologias móveis ganham espaço nas sociedades e mostram-se eficazes e economicamente viáveis para uso nas operações da Logística Humanitária, concernente as comunicações de risco.

No entanto mais aplicativos devem ser pensados, ou aprimorados, de forma que possibilitem um uso eficiente e assertivo no antes, durante e após eventos de desastre.

Mas é importante ressaltar, assim como já foi citado no texto, para uma boa comunicação de risco, será importante a elaboração de protocolos claros a serem seguidos pelos agentes de ajuda humanitária, de forma a se proporcionar uma comunicação eficaz e segura, evitando ambiguidades, mal entendidos e informações falsas.

\section{REFERÊNCIAS}

ABNT. Gestão de Riscos - Princípios e diretrizes. NBR ISO 31000. Associação Brasileira de Normas Técnicas, 2009.

BRASIL Lei no 12.608 de 10 de abril de 2012. Institui a Política Nacional de Proteção e Defesa Civil - PNPDEC; dispõe sobre o Sistema Nacional de Proteção e Defesa Civil - SINPDEC e o Conselho Nacional de Proteção e Defesa Civil - CONPDEC; autoriza a criação de sistema de informações e monitoramento de desastres; altera as Leis nos 12.340, de 1o de dezembro de 2010, 10.257, de 10 de julho de 2001, 6.766, de 19 de dezembro de 1979, 8.239, de 4 de outubro de 1991, e 9.394, de 20 de dezembro de 1996; e dá outras providências. Diário Oficial da União, Brasília, DF: Poder Executivo, 2012.

CEPED/UFSC (2014) Capacitação básica em defesa civil / [textos: Janaína Furtado. Marcos de Oliveira; Maria Cristina Dantas; Pedro Paulo Souza; Regina Panceri]. - 5. ed. Disponível em: http://www.ceped.ufsc.br/wp-ontent/uploads/2013/01/Livro_DefesaCivil_5ed-DiagramadoCompleto-online.pdf." Acesso em: 27/03/2016.

CISCO GLOBAL HOME PAGE. Cisco Annual Internet Report, 2018-2023, 2020. Disponível em: $<$ https://www.cisco.com/c/en/us/solutions/collateral/executive-perspectives/annual-internetreport/white-paper-c11-741490.html>. Acesso em: 18/09/2020.

COLlinS, M., NEVILLE, K., HYNES, W., MADDEN, M. Communication in a disaster - the development of a crisis communication tool within the S-HELP project. Journal of Decision Systems, v. 25, p. 160-170, 2016.

COSTA, F. G.; FLAUZINO, R. F.; NAVARRO, M. B. M. A.; CARDOSO, T. A. O. Abrigos temporários em desastres: a experiência de São José do Rio Preto. Saúde Debate, v.41, p.327-337, 2017.

DA SILVA, A. M.; STEINBERGER-ELIAS, M. B. II Encontro Internacional Tecnologia, Comunicação e Ciência Cognitiva Universidade Metodista de São Paulo - 03 e 04 de dezembro de 2015. p. 1-18, 2015.

FOWLER, M. UML Essencial: um breve guia para a linguagem padrão de modelagem de objetos / Martin Fowler; Trad. João Tortello - $3^{\mathrm{a}}$ Ed. - Porto Alegre: Bookman, 2005. 


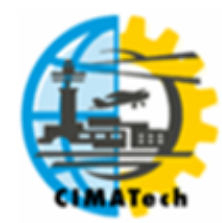

FreitAS, C. M.; De CARVAlhO, M. L.; XIMENES, E. F.; ARRAES, E. F.; GOMES, J. O. Vulnerabilidade socioambiental, redução de riscos de desastres e construção da resiliência: lições do terremoto no Haiti e das chuvas fortes na Região Serrana, Brasil. Ciênc. saúde coletiva [online], vol.17, n.6, p.1577-1586, 2012.

HASELKORN, M.; WALTON, R. The role of information and communication in the context of humanitarian service. IEEE Transactions on Professional Communication, v. 52, n. 4, p. 325-328, 2009.

HENRIKSEN, H. J.; ROBERTS, M. J.; VAN DER KEUR, P.; HARJANNE, A.; EGILSON, D.; ALFONSO, L. Participatory early warning and monitoring systems: A Nordic framework for webbased flood risk management. International Journal of Disaster Risk Reduction, v. 31, n. January, p. 1295-1306, 2018.

JOHN WELLINGTON, J.; RAMESH, P. Role of Internet of Things in disaster management. Proceedings of 2017 International Conference on Innovations in Information Embedded and Communication Systems, v. 2018, p. 1-4, 2018.

JOSEPH, J. K.; DEV, K. A.; PRADEEPKUMAR, A. P.; MOHAN, M. Big data analytics and social media in disaster management. In: Integrating Disaster Science and Management. Global Case Studies in Mitigation and Recovery. Elsevier Inc., 2018.

LIMA, A. S. A. de; SCHMIDT, A. E. F.; NOGUEIRA, R. R.; OLIVEIRA, P. C. F. de. Aplicativo colaborativo para alerta de vulnerabilidade a alagamentos e enchentes no Vale do Itajaí. Anais do IX Workshop de Computação Aplicada a Gestão do Meio Ambiente e Recursos Naturais, 2018

LORENZO, E. M. A Utilização das Redes Sociais na Educação. $3^{\mathrm{a}}$ ed., Rio de Janeiro, Clube de Autores, 2013.

LV, X.; LIAO, Y.; DENG, L. Natural Disaster Emergency Rescue System Based on the Mobile Phone's High-Precision Positioning. 3rd IEEE International Conference on Image, Vision and Computing, ICIVC 2018, p. 797-801, 2018.

MARTINS, M. H. da M.; SPINK, M. J. P. O uso de tecnologias de comunicação de riscos de desastres como prática preventiva em saúde. Interface (Botucatu), v. 19, n. 54, p. 503-514, 2015.

MURAYAMA, Y., YAMAMOTO, K. Research on disaster communications. Second IFIP TC 5 DCITDRR International Conference, v. 516, p. 1-11, 2019.

NASCIMENTO JÚNIOR, V. F. Estudo Sistemático Sobre Testes de Usabilidade Realizados em Aplicativos Móveis. Trabalho de Conclusão de Curso de Graduação em curso de Sistemas de Informação - Universidade Federal de Pernambuco, Recife, 2017. Disponível em: $<$ https://www.cin.ufpe.br/ tg/2017-2/vfnj-tg.pdf $>$. Acesso em: 15/09/2019. 


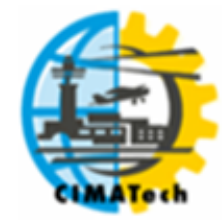

OLIVEIRA, E. L. DE A.; ROBAINA, L. E. DE S. Gerenciamento de Áreas de Risco em Cidades Brasileiras: Projetos e Programas. Ciência e Natura, v. 37, n. 3, p. 366-384, 2015.

OLIVEIRA, G. M.; SANTOS, L. F. USO DE APLICATIVOS PARA DISPOSITIVOS MÓVEIS NO PROCESSO DE EDUCAÇÃO EM SAÚDE: reflexos da contemporaneidade. Revista Observatório, v. 4, n. 6, p. 826-844, 2018.

REUTER, C.; KAUFHOLD, M. A.; SPIELHOFER, T.; HAHNE, A. S. Social media in emergencies: A representative study on citizens' perception in Germany. Proceedings of the ACM on HumanComputer Interaction, v. 1, n. CSCW, 2017.

SIMON, T.; GOLDBERG, A.; ADINI, B. Socializing in emergencies - A review of the use of social media in emergency situations. International Journal of Information Management, v. 35, n. 5, p. 609-619, 2015.

TYSHCHUK, Y.; WALLACE, W. A. Modeling Human Behavior on Social Media in Response to Significant Events. IEEE Transactions on Computational Social Systems, v. 5, n. 2, p. 444-457, 2018.

UNISDR, United Nations International Strategy for Disaster Reduction (2015) Sendai Framework for Disaster Risk Reduction 2015-2030. UN world conference on disaster risk reduction, 2015. March 14-18, Sendai, Japan. Geneva: United Nations Office for Disaster Risk Reduction; 2015. Disponível em: < https://www.preventionweb.net/files/43291_sendaiframeworkfordrren.pdf. Acesso em: 03/08/2020.

VAHIDNIA, M. H.; HOSSEINALI, F.; SHAFIEI, M. Crowd source mapping of target buildings in hazard: the utilization of smartphone technologies and geographic services. Applied Geomatics, v. 12, p. 3-14, 2020.

VYNCKE, B.; PERKO, T.; VAN GORP, B. Information Sources as Explanatory Variables for the Belgian Health-Related Risk Perception of the Fukushima Nuclear Accident. Risk Analysis, v. 37, n. 3, p. 570-582, 2017. 